\title{
The Araucaria project - improving stellar distance indicators
}

\author{
Grzegorz Pietrzyński ${ }^{1,2}$, Wolfgang Gieren ${ }^{1}$ \\ ${ }^{1}$ Departamento de Fisica, Universidad de Concepción, Casilla 160-C, \\ Concepción, Chile \\ ${ }^{2}$ Warsaw University Observatory, Aleje Ujazdowskie 4, 00-478, \\ Warszawa, Poland
}

\begin{abstract}
We present first results of an optical wide field imaging survey of the galaxies NGC 300, NGC 6822, WLM and Phoenix, performed as part of the Araucaria Project, a long-term observational program recently initiated at the Universidad de Concepción. Based on our data, a large number of new Cepheids in these galaxies has been found. Our photometric databases were also used to select blue supergiant candidates in these galaxies, and to search for the photometric variability of these stars, as well as for a number of side projects, such as the search of other types variable stars and $\mathrm{OB}$ associations in these galaxies.
\end{abstract}

\section{Introduction}

We have recently started on a long-term observational program called the Araucaria Project (Gieren et al. 2001), which aims to check on the systematics and true capabilities of several stellar methods of distance measurement, using our basic strategy to apply these stellar standard candles on a common sample of galaxies with widely different environmental properties, and to compare the results. This should finally lead to a clear picture about the true capabilities of the different methods and their dependence on environmental properties such as metallicity and age, and will help us to select and calibrate those techniques which give the most accurate results. In our project, we are analyzing the potential for distance measurement of the following standard candles: Cepheids, blue supergiants, Terminal Red Giant Branch (TRGB), red clump stars, and RR Lyrae stars.

To secure the high quality data for these objects in our target galaxies that is needed for our research, we are using a variety of telescopes and instruments. The first logical step that needs to be carried out is the performance of extensive multi-epoch Wide Field optical surveys in our target galaxies. Here we present first results from such surveys recently finished for NGC 300, NGC 6822, WLM and Phoenix. 


\section{Observations}

Wide field imaging of the Sculptor Group spiral NGC 300 was performed with the 2.2-m MPI telescope at La Silla, ESO. The Local Group galaxies were monitored with the 1.3-m Warsaw telescope installed at the Las Campanas Observatory. Both telescopes were equipped with Wide Field imagers $(8 \mathrm{k} \times 8 \mathrm{k}$ mosaic detectors) having large fields of view (about $35^{\prime} \times 35^{\prime}$ ) with excellent scales of about $0.25^{\prime \prime} / \mathrm{pix}$, allowing to cover each of the galaxies with a single shot.

The observations were conducted over $0.5-2 \mathrm{yr}$ through $B V I$ filters. At least forty, and up to a hundred observations were secured in each filter for each galaxy.

\section{First results}

\subsection{Cepheids}

In NGC 300, we discovered 129 Cepheids (Pietrzyński et al. 2002). Our search should be fairly complete for Cepheids with periods longer than approximately $12 \mathrm{~d}$. The period-luminosity relation in the $V$ band obtained from our data is shown in Fig. 1. Unfortunately, the strong fringing present in the $I$-band images obtained with the ESO 2.2-m WFI did not allow us to obtain accurate mean magnitudes of the Cepheids in this band. We are currently obtaining new $I$-band mosaic images of NGC 300 with the LCO Warsaw telescope which will be used in combination with the $V$-band images to obtain an accurate determination of the distance of NGC 300 from the reddening-free Wesenheit PL relation.

In NGC 6822, we found about 80 Cepheid candidates from our images. The resulting Wesenheit index period-luminosity relation is presented in Fig. 2. Its slope of $3.24 \pm 0.06$ is in excellent agreement with previous studies for the LMC and SMC (Udalski et al. 1999), and IC 1613 (Udalski et al. 2001), indicating that the slope of the PL relation in this band does not depend on metallicity in the very broad metallicity range from -0.3 to -1.0 dex. The difference between the $I$-band brightness of Cepheids with a period of $10 \mathrm{~d}$, and the $I$-band magnitude of the TRGB was found to be $0.69 \mathrm{mag}$, again in excellent agreement with results obtained for the Magellanic Clouds and IC 1613 (Udalski et al. 2001). This suggests that the zero point of the $I$-band PL relation for Cepheids in this metallicity range does not significantly depend on metallicity, either.

Previous searches for Cepheids in WLM (Sandage \& Carlson 1985) were performed from very inhomogeneous photographic data. From our WF survey we expected to find a significant number of new, previously undetected Cepheids in this galaxy. Indeed, by detecting 30 Cepheids we doubled the number of these stars now known in WLM. However, we found just one long period Cepheid, with a period of about $54 \mathrm{~d}$. All the remaining Cepheids have periods shorter than $11 \mathrm{~d}$.

From about 60 images obtained over 7 months we could not detect any Cepheid in the Phoenix galaxy, in contrast to the claim in Caldwell et al. (1988). Our Cepheid search for this galaxy should be complete for stars with periods longer than $3 \mathrm{~d}$. 


\subsection{Blue supergiants}

Our optical images served to select candidates for blue supergiant stars in our target galaxies using selection criteria based on the expected magnitudes and colors of these objects. From our multi-epoch photometric data it is also possible to check if the distance determination with these objects from the Wind Momentum-Luminosity Relationship (Kudritzki \& Puls 2000) and the FluxWeighted Gravity-Luminosity Relation (Kudritzki et al. 2003) is significantly affected by their photometric variability. To answer this question we analyzed the $V$-band light curves of a spectroscopically confirmed sample of blue supergiants in NGC 300 (Bresolin et al. 2002a). As a result, we found that most of our targets do show small changes of their brightness, but of a size small enough that the effect on the distance determination with these two methods is negligible (Bresolin et al. 2003).

\subsection{Sub-projects}

Our photometric data collected in the course of the wide field imaging of our target galaxies represent an excellent base for many different sub-projects, such as the study of the variable star content of our galaxies, the search for and analysis of OB associations (Pietrzyński et al. 2001), or a detailed analysis of particularly interesting objects like the Ofpe/WN9 star discovered in NGC 300 (Bresolin et al. 2002b).

Acknowledgments. Support from the Chilean Center for Astrophysics FONDAP 15010003 and the Polish KBN grant 2P03D02123 is acknowledged.

\section{References}

Bresolin, F., Gieren, W., Kudritzki, R., Pietrzyński G., Przybilla, N. 2002a, ApJ, 567, 277

Bresolin, F., Kudritzki, R., Najarro, F., Gieren, W., Pietrzyński G., 2002b ApJ, 577, L107

Bresolin, F., Pietrzyński, G., Gieren, W., Kudritzki, R., Przybilla, N., Fouqué, P. 2003, ApJ, in press

Caldwell, N., Schommer, R.A., Graham, J.A. 1988, BAAS, 20, 1084

Gieren, W., Geisler, D., Richtler, T., Pietrzyński, G., Dirsch, B. 2001, Messenger, 106, 15

Kudritzki, R., Bresolin, F., Przybilla, N. 2003, ApJ, 582, 83

Kudritzki, R., Puls, J. 2000, ARA\&A, 38, 613

Pietrzyński, G., Gieren, W., Fouqué, P., Pont, F. 2001, A\&A, 371, 497

Pietrzyński, G., Gieren, W., Fouqué, P., Pont, F. 2002, AJ, 123, 789

Sandage, A., Carlson, G., 1985, AJ, 90, 1464

Udalski, Szymański, M., Kubiak, M., Pietrzyński, Soszyński, I., Woźniak, P., Żebruń, K. 1999, Acta Astron., 1999, 49, 201

Udalski, A., Wyrzykowski, Ł., Pietrzyński, G., Szewczyk, O., Szymański, M., Kubiak, M., Soszyński, I., Żebruń, K. 2001, Acta Astron., 51, 221 


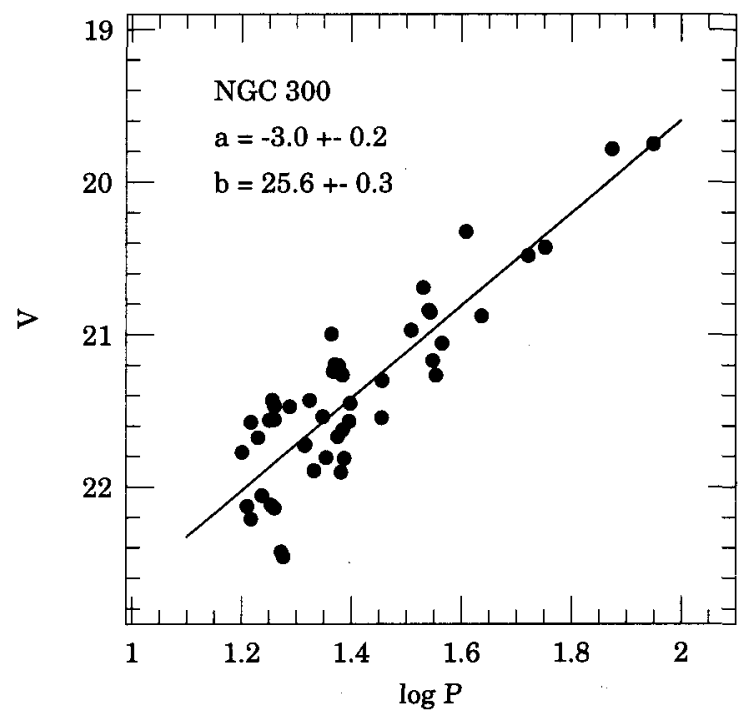

Figure 1. $\quad V$-band Period-Luminosity relation for Cepheids in NGC 300.

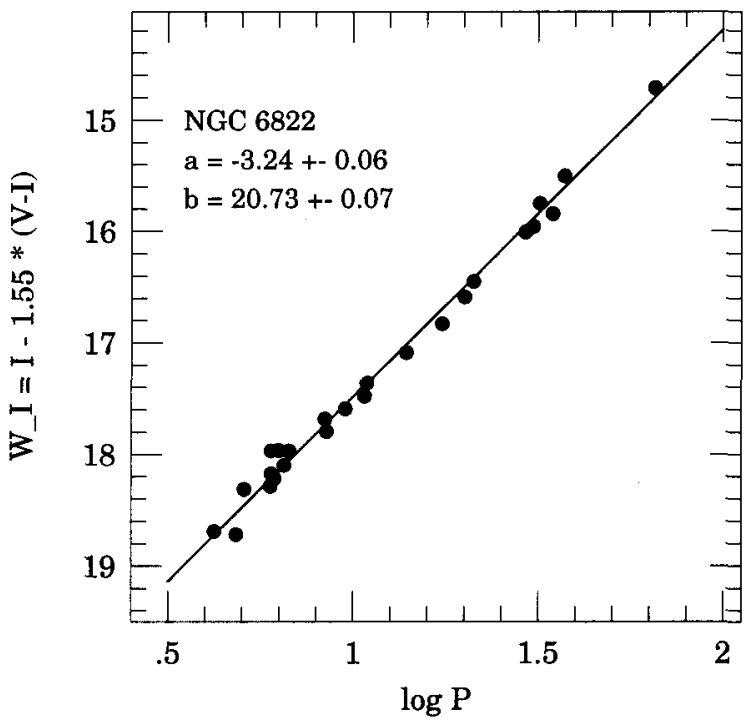

Figure 2. Wesenheit index versus period for Cepheids in NGC 6822. 